\title{
Comportamiento de la accidentalidad en una empresa metalmecánica en Cartagena, Colombia
}

\author{
Behavior of accidents an engineering company in Cartagena, Colombia
}

Elías Alberto Bedoya Marrugo

\section{Resumen}

Objetivo. Establecer el comportamiento de la accidentalidad de una empresa del sector metalmecánico en la periferia de la ciudad de Cartagena, durante el periodo 2014. Métodos. Revisión de datos de accidentalidad de una empresa metalmecánica durante el años 2014, junto a una discriminación de los tipos de accidente y características de los cargos, clase y tipo de vinculación de los afectados, tipos de lesiones, mecanismos de la lesión y accidentes incapacitantes, accidente por cargo, antigüedad y lugar de ocurrencia. Resultados. Se encontró un total de 36 accidentes laborales, en el cual las prevalencias fueron entre otras: cargos afectados ayudante de planta con 33\%, aprendiz 22\%, $75 \%$ de los afectados son de la empresa, mientras el $25 \%$ restante pertenecen a contratistas, siendo el mes de octubre y abril los más accidentados con un 22.2\% respectivamente, las heridas en extremidades con $66 \%$ son el tipo de lesión más común, seguido por el aplastamiento con un $27 \%$. La accidentalidad reportada en esta investigación se presentó de forma importante en los trabajadores en los cargos de aprendiz y ayudante de planta, y afectó principalmente a las extremidades superiores e inferiores.

Palabras clave: accidentalidad, industria metalmecánica, lesión, incapacidad física.

\begin{abstract}
Objective. To establish the accident rate behavior in a company in the metalworking sector in the periphery of the city of Cartagena in 2014. Method. Reviewing of accident data from a metal working company in 2014, together with a classification of the types of accidents and characteristics of the positions, class and type of the affected, type of injury, injury mechanism, disabling accidents, accidents by position, seniority, and place of occurrence. Results. A total of 36 accidents were found in which the prevalence of affected positions, among others, were: plant assistants (33\%) and trainees (22\%). 75\% of those affected are company employees, while the remaining $25 \%$ are contractors. October and April reported the majority of accidents (22.2\%). Limb wounds (66\%) are the most common type of injury, followed by crushing with $27 \%$. The accidents reported in this research significantly affected trainees and plant assistants, and it mainly affected the upper and lower extremities.
\end{abstract}

Keywords: accident rate, disability, industry, injury, metalworking.

Coordinador de investigación, Fundación Universitaria Tecnológico Comfenalco, Facultad de ingeniería, Programa de Tecnología en seguridad e higiene ocupacional.

Correspondencia: eabedoya8@gmail.com

Elías Alberto Bedoya Marrugo Orcid: 0000-0002-2931-9600

Recibido: 10/10/2015 Aceptado: 15/105/2015 


\section{Introducción}

Avances tecnológicos que tuvieron inicio con la bombilla eléctrica y posteriormente con la reinvención del acero son determinantes para la industria a partir de la segunda mitad del Siglo XIX y decisivos para apalancar la segunda revolución industrial. Acero y electricidad realimentaron la industria metalmecánica permitiendo la aparición de nuevas y mejores formas de explotación que influyeron en los sectores textil, ferroviaria y de armamento hasta nuestros días (1). Es necesario resaltar que en la industrias metalmecánicas existe una gran diversidad de riesgos, dentro de los cuales se encuentran: los de índole física (ruido, iluminación, temperatura), químicos, psicosociales, ergonómicos y otros (2).

Las consecuencias de los accidentes de trabajo citadas por Keller y Meza son interpretadas como el sufrimiento humano, dolor, preocupación familiar, además de pérdidas de tiempo y dinero, que además repercuten en gastos de atención médica, quirúrgica, farmacéutica, pago del sueldo por incapacidad, indemnizaciones, pago de prótesis o tratamientos posteriores, siendo éstos, costos que la empresa tiene que cubrir $(3,4)$. Los aspectos económicos de los accidentes guardan estrecha relación con los de su prevención; cuanto más dinero se gasta en la prevención de los accidentes, menor será el dinero en los gastos realizados en la consecuencia de los accidentes. Lo ideal es prevenir y, esto a su vez economizará el costo de los accidentes (5).

Con relación al análisis de causa-efecto de la seguridad laboral, la principal causa por la cual se presentan accidentes o incidentes de trabajo en las empresas metalmecánicas, se debe a la falta de compromiso de los empleados con el uso de los elementos de protección personal, pues la empresa cumple con su responsabilidad de suministrarlos, pero el $87.5 \%$ de los trabajadores no utilizan los elementos de protección personal (EPP) necesarios ni toman las debidas precauciones, generando además actos inseguros. Un $62,5 \%$ de los accidentes es generado por golpes sufridos en el trabajo (golpeado por o contra), esto se debe especialmente al descuido de los trabajadores y a la mala ubicación de objetos en la planta (6).

La metalmecánica es una de las actividades económicas que muestra una evolución constante en sus modos de producción, entre las labores que se desarrollan en dicha actividad se presentan una gran cantidad y diversidad de riesgos profesionales que repercuten a corto, mediano y largo plazo en la salud del trabajador (7). A corde con las premisas de las administradoras de riesgos manejar un programa de cultura de salud ocupacional y autocuidado para trabajadores informales del sector metalmecánico ha sido una preocupación de alto interés en administraciones locales relacionadas con el sector metalmecánico, afectado por la accidentalidad de los últimos años (8).

Para la Organización Internacional del Trabajo (OIT), en el mundo mueren al año 2,2 millones de personas a causa de accidentes y enfermedades relacionadas con actividades laborales, se registran 270 millones de accidentes de trabajo no mortales y 160 millones de casos relacionados con enfermedades profesionales, cifras éstas últimas que, en un tercio, conllevan a la pérdida de cuatro días laborales por persona afectada (9). Como afirman Pérez y Col 2007 en relación al trabajo en la industria metalmecánica lleva a los trabajadores a exponerse a diferentes riesgos físicos, químicos biológicos, psicosociales y ergonómicos que si no se controlan a tiempo pueden ocasionar accidentes y enfermedades laborales, todos estos riesgos son descritos cuantitativamente pero no se toma en cuenta al momento de realizar una evaluación de puesto de trabajo la percepción subjetiva de los actores participantes, siguiendo con este hallazgo se encontró que de igual forma son los actos inseguros los mayores desencadenantes de accidentes en la industria metalmecánica (10).

Zamorano y Colaboradores consideran que el origen de los accidentes pueden ser los actos y las condiciones inseguras, los actos inseguros son los que 
estadísticamente representan mayor responsabilidad en la ocurrencia (11). Los estudios nacionales sobre cambios sociales, ocurridos en relación con el paradigma tecnológico basado en microelectrónica, son recientes. Los relacionados de modo específico con MHCN y CNC, afirman que hacia 1983 la difusión de esta tecnología en la metalmecánica colombiana era prácticamente nula, lo que indica que el avance tecnológico en métodos, técnicas y prevención de accidentes no se había generado en el país, lo que demuestra que en la actualidad en este campo tan activo de la economía colombiana no ha avanzado significativamente (12). Si se toma en cuenta que el costo de la accidentalidad en Colombia podría ser cercano al 5.5\% del PIB del ańo esto representa más de $\$ 7.96$ billones cada año, que se podrían invertir en otros aspectos prioritarios para el país, por ejemplo en alrededor de $410.000 \mathrm{vi-}$ viendas de interés social, y cerca de 2'500.000 becas estudiantiles (13).

La Organización Internacional del Trabajo (OIT) considera el accidente de trabajo como el resultado de una cadena de acontecimientos en la que "algo" ha funcionado mal y no ha llegado a buen término, estos acontecimientos que acaban produciendo lesiones se deben a ciertos factores existentes en los lugares de trabajo, lo que lleva a concluir que la magnitud del problema debe determinarse en función de la existencia y frecuencia de tales factores (14). La principal forma de accidente en el sector del metal es debida a golpes por objetos o herramientas (22,2\% del total de accidentes en jornada de trabajo) y, en segundo lugar, a sobreesfuerzos $(20,5 \%)$, dando lugar a lumbalgias en un 8,8\% de los casos. Estos dos tipos de accidentes, juntamente con las proyecciones de fragmentos o partículas, llegan a representar prácticamente el $60 \%$ del total de los accidentes del sector (15).

\section{Materiales y métodos}

En este estudio, de corte descriptivo y transversal, se hace una evaluación de datos estadísticos con la revisión de datos de accidentalidad en una empresa metalmecánica en el año 2014, identificando las principales características de los eventos mediante la revisión del reporte de 36 accidentes ocupacionales en el ańo 2014 iniciando por tipos de accidente, clase de afectados, cargos de los accidentados, tipo de vinculación de los afectados, tipos de lesiones, mecanismos de la lesión y accidentes incapacitantes, accidente por cargo, antigüedad y lugar de ocurrencia.

Se realizó una revisión amplia y profunda de la literatura relacionada con la seguridad laboral y la productividad del sector metalmecánico; posteriormente, se diseñó instrumento estructurado que permitiera recopilar información efectiva en lo que concierne a la caracterización de los procesos de la empresa, análisis de los riesgos e incidencia de la seguridad laboral e indicadores de accidentalidad (frecuencia, incidencia y gravedad). Se incluyeron trabajadores reportados entre los 36 accidentes registrados, además con los accidentes generados en el año 2014, se utilizó como criterio de exclusión el no pertenecer a empresa o no estar incluido entre los trabajadores accidentados en la empresa.

Se tomó como principal fuente de información a los reportes de accidentes realizados durante el año 2014, registrando de igual forma en el historial ocupacional de los trabajadores involucrados. Datos profesionales: área, cargo, antigüedad en la empresa. Se acudió a la oficina de recursos humanos donde se obtuvieron los registros y datos particulares de los afectados, se organizó la información y tabularon los datos relacionados por característica específica. Después se realizó la digitación de datos en hoja de cálculo Excel, procesamiento de datos y análisis de información mediante herramientas estadísticas y posteriormente presentación de datos en tablas y gráficos. Para el análisis descriptivo de los datos, se hizo uso de la computadora utilizando herramientas de estadística descriptiva. 


\section{Resultados}

Se manejaron 36 accidentes de trabajo en la empresa metalmecánica, el 100\% de los accidentados son hombres, eventos de los cuales el 75\% (27 de 36) pertenecen a eventos ocurridos a trabajadores de la empresa contratante, el restante 25\% (9 de 36) ocurrieron a contratistas de la empresa principal.

Tabla 1. Características sociodemográficas de la población objeto de estudio

\begin{tabular}{|c|}
\hline $\begin{array}{l}\text { Características Sociodemográficas } \\
\qquad \begin{array}{c}\mathbf{n}=36 \\
\text { VARIABLE } \mathbf{n}(\%)\end{array}\end{array}$ \\
\hline Edad promedio 27 años (DS 6,8) \\
\hline Masculino $36(100 \%)$ \\
\hline Estrato $1.23(63,8 \%)$ \\
\hline Estrato $2.10(27,7 \%)$ \\
\hline Estrato $3.3(8,3 \%)$ \\
\hline casado $14(38,8 \%)$ \\
\hline Soltero $13(36,11 \%)$ \\
\hline Unión libre 9 ( $25 \%$ ) \\
\hline
\end{tabular}

Conforme a la Tabla 1, de características sociodemográficas se identificó un promedio de edad de los accidentados de 27 ańos, (DS 6,8). El accidentado de mayor edad tiene 45 años al momento de evento, mientras el más joven reportó 18 años. Entre los accidentados la edad con más frecuencia es los 25 años con 5 trabajadores con esta edad, el 63.8\% (23 de 36) pertenecen al estrato 1 , mientras el $27.7 \%$ al estrato 2 y $8.3 \%$ al estrato 3. El $38.8 \%$ de las accidentados (14 de 36) son casados, seguidos por los solteros con $36.11 \%$ (13 de 36), mientras 9 de los afectados, es decir $25 \%$ del total viven en unión libre. La escolaridad encontrada entre los afectados por accidentes en esta investigación indica que 50\% (18 de 36) de los trabajadores son bachilleres, un $25 \%$ (9 de 36) tienen estudios incompletos en la secundaria y otro $25 \%$ tienen estudios de básica primaria.
Tabla 2. Aspectos ocupacionales

\begin{tabular}{c} 
Aspectos ocupacionales \\
$\mathbf{n}=\mathbf{3 6}$ \\
VARIABLE n (\%) \\
Ayudante $17(47,2 \%)$ \\
Aprendiz $8(22,2 \%)$ \\
\hline Operador - Soldador $8(22,2 \%)$ \\
Mecánico-Supervisor $3(8,3 \%)$ \\
\hline Trabajador de la empresa $27(75 \%)$ \\
\hline Contratista Externo $9(25 \%)$ \\
Menos de 1 mes $15(41,6 \%)$ \\
Menos de 1 año $18(50 \%)$ \\
Más de 1 año $3(8,3 \%)$
\end{tabular}

En la Tabla 2, con referencia a aspectos ocupacionales, se observa que la antigüedad promedio de los afectados en la empresa al momento del accidente fue de 7 meses. Los cargos afectados por accidentes de trabajo son ayudante $47.2 \%$ (17 de 36 ); aprendiz y operador-soldador $22.2 \%$ (8 de 36 ) respectivamente, mientras el cargo de mecánico supervisor con 8.3\% (3 de 36) obtuvo la menor prevalencia. Resalta que el 75\% (27 de 36) de los accidentes ocurrieron a trabajadores de la empresa, mientras 25\% (9 de 36) eran contratistas. De los afectados 50\% (18 de 36) fueron trabajadores que tenían menos de un año laborando en la empresa, mientras 15 de estos (41.6\%) tenían menos de un mes y sólo $8.3 \%$ (3 de 36) tenían más de un año de labores en la organización.

Tabla 3. Características del accidente

Características del accidente
$\mathbf{n}=36$
VARIABLE $\mathbf{n}(\%)$
Área de Producción (94,4\%)
Tipo de Lesión
Herida $24(66,6 \%)$
Golpe $10(27,7 \%)$
Otro $2(5,5 \%)$
Agente Causal
Materiales $10(27,7 \%)$
Herramientas 24 (66,6\%)
Otro $2(5,5 \%)$




\section{Mecanismos de Lesión}

Materiales y maquinas 15 (41,6\%)

Atrapamientos 10 (27,7\%)

Caída de objetos 9 (25\%)

Contacto con sustancias 2(5,5\%)

Accidente incapacitante 23 (63,8\%)

Accidente sin incapacidad 13 (36,1\%)

En la Tabla 3 de características del accidente se observa que en el área de producción la ocurrencia de los accidentes fue $94.4 \%$ (34 de 36) y el restante $5.5 \%$ ( 2 de 36 ) tuvo como escenario las oficinas de administración. Las heridas de diverso tipo con 66\% (24 de 36), golpe inespecífico 27.7\%(10 de 36) y otras lesiones con $5.5 \%$ ( 2 de 36 ) son la tipificación de las lesiones encontradas en esta investigación que afectaron principalmente a las extremidades superiores (manos y brazos) siendo estas zonas las más afectadas.

Entre los agentes causales de los accidentes esta investigación se destacan las herramientas manuales con un $66.6 \%$ (24 de 36), los materiales con un $27.7 \%$ (10 de 36), y luego otros agentes con 5.5\% ( 2 de 36$)$ fueron identificados. Dentro de los me-

Indicador

Formula

Cantidad

HHT $=8$ Horas X 6 días a la Semana X 4,29 (Factor de conversión de semana a mes) X 12 meses (año) X número conversión de semana a mes) $X 12 \mathrm{~m}$
de trabajadores canismos que originaron los accidentes sobresalen las maquinas $41.6 \%$ (15 de 36), los atrapamientos $27.7 \%$ (10 de 36), la caída de objetos con 25\% (9 de 36) y el contacto con sustancias 5.5\% (2 de 36).

De los eventos reportados en el año 2014, se presentó un nivel de incapacidades por accidentalidad del 63\% (23 de 36), mientras el restante 36.1\% (13 de 36) no generaron incapacidad. El promedio de días de incapacidad entre los accidentados fue de 6 días. Se encontró un caso de accidentado que hasta la fecha no se ha reincorporado a labores por encontrarse aun incapacitado. El mes de abril fue el más accidentado y generó más incapacidades en esta empresa en el 30.5\% de los eventos.

Los datos obtenidos en esta investigación han permitido diseñar una tabla que contiene las principales observaciones para desarrollar indicadores como los índices de frecuencia de accidentes de trabajo, índice de severidad de accidentes e índice de lesiones incapacitantes, dichos indicadores se presentan e interpretan a continuación:

Tabla 4. Algunos indicadores del trabajo de observación
Horas hombre trabajadas
165559,8

Días perdidos por incapacidad de Días de incapacidad otorgados a los trabajadores accidenorigen ocupacional tados

51
Índice de frecuencia de accidente de trabajo

Total de accidentes en año X K / HHT año

21,7

Índice se severidad de accidente de trabajo
No.de días perdidos y cargados por AT en el año X K /

HHT año
30,8

0,6

Índice de lesión incapacitante de accidente de trabajo 
Indicadores 2014. La accidentalidad registrada demuestra que la afectación por este fenómeno alcanza niveles altos, si se tiene en cuenta el número de horas hombre trabajadas, de igual forma, el nivel de días perdidos puede considerarse como lesivo para la empresa, debido a los costos adicionales en que habría que incurrir para suplir las ausencias de las personas lesionadas durante los días de ausencias justificadas por accidentes relacionados al trabajo, ingresando personal nuevo que requiere inducción y al no poder igualar su nivel de producción y al mantenerse esta tendencia la empresa enfrentaría dificultades para suplir el déficit provocado por los accidentes, al entenderse que cada 0.6 accidentes ocurridos lesiona e incapacita a los trabajadores involucrados.

\section{Discusión}

De acuerdo a lo informado por Nathan que resalta al campo de la metalmecánica como uno de los más ricos en riesgos laborales, este estudio encontró factores desencadenantes de accidentalidad que para el caso puntual han tenido amplia injerencia en la productividad de la empresa objeto de estudio (2). Teniendo en cuenta las cifras entregadas por este estudio destaca que la accidentalidad de los trabajadores informales del sector metalmecánico es una seria preocupación que cobra un alto interés en los últimos años.

En cuanto a lo referido por los autores Meza y Keller $(3,4)$ sobre las consecuencias de los accidentes de trabajo, se encontraron coincidencias en lo relacionado con las pérdidas de tiempo y dinero, al desplazar la producción de un trabajador por la atención médica y el pago del sueldo por incapacidad donde la empresa estudiada alcanzó a perder cerca de 51 días por concepto de incapacidades en accidentes de trabajo (5). Al remitirse a las causas de los accidentes en estudio se afirma que son los elementos y materiales utilizados los que generaron los accidentes enmarcados como actos inseguros $\mathrm{Ha}$ llazgos similares a los reportados por Zamorano y Cols quienes encontraron en su estudio que los actos inseguros son estadísticamente los de mayor responsabilidad en la ocurrencia de accidentes (8), así mismo, se encontró que igual a Cortes (2007), son los actos inseguros los mayores desencadenantes de accidentes en la industria metalmecánica (9).

Se evidenció en esta investigación que en la empresa evaluada el manejo de las estadísticas fue inexistente, siendo el no registro y subregistro de los accidentes y enfermedades relacionadas con el trabajo, entre los eventos que requieren la intervención de los organismos de control liderados por el Ministerio de Salud y Protección Social (12).

Como conclusión, la accidentalidad reportada en esta investigación afectó de forma importante a los trabajadores en los cargos de aprendiz y ayudante de planta, como también afectó principalmente a las extremidades superiores e inferiores (pies y manos). Detallando el comportamiento de esta empresa, en relación a los eventos de accidentes, se logra determinar que es el subregistro uno de los responsables de una mala determinación. Teniendo en cuenta los hallazgos y reflexiones generadas en este estudio se establecieron las siguientes recomendaciones en cuanto a la accidentalidad en una empresa metalmecánica.

- Desarrollar un programa de inducción capacitación y entrenamiento para el personal nuevo, y en entrenamiento, debido a que este grupo (aprendices y ayudantes) fueron los más accidentados de la investigación.

- Emplear un manual de buenas prácticas de seguridad en el cual se consignen las normas y protocolos de seguridad por las que deben regirse todos los empleados de la empresa sin excepción para así garantizar un entorno donde la seguridad se considere una cultura y no una obligación.

- Introducir a la personal en el manejo practico de los riesgos donde se les retroalimente de forma cotidiana sobre el resumen del panorama de 
riesgos de la empresa, principales riesgos, vulnerabilidad y amenazas principales.

- Realizar visitas de inspección a cada área de la empresa en especial a las zonas del patio de labores donde se han ocurrido los accidentes, teniendo como principal objetivo la identificación periódica de los riesgos existentes, con el fin de identificar, ubicar y jerarquizar factores de riesgo presentes en los distintos puestos de trabajo de la empresa con el fin de implementar las medidas de mitigación que permitan crear condiciones de higiene y seguridad adecuadas para el desarrollo del trabajo que eviten la generación de situaciones peligrosas.

\section{Referencias}

1. Nathan R. Tecnología y Economía. Barcelona: Gustavo Gili; 1979.

2. Castillo S, Torres López T. Percepción de riesgos laborales en trabajadores de industrias metalmecánicas. Instituto de Investigación en Salud Ocupacional. 2011;2(2):15-17.

3. Keller's Official OSHA Safety Handbook. Winsconsin: J.J. Keller \& Associates; 1995.

4. Meza S. Higiene y seguridad industrial. Ciudad de México DF: Instituto Politécnico Nacional.; 1998.

5. Sánchez S. Higiene y Seguridad Industria. Ciudad de México: Editorial; 2010.

6. Morelos J, Fontalvo Herrera T. Caracterización y análisis del ries- go laboral en la pequeńa y mediana industria metalmecánica en Cartagena. Revista Soluciones de Postgrado EIA. 2013;10(1):1337.

7. Sánchez J, Castillo S, Rodríguez B. Accidentes de trabajo en una empresa ferroviaria. Revista de Medicina y Seguridad del Trabajo. 2005;51(199):15-25.

8. Zamorano González B, Parra Sierra V, Peña Cárdenas F. Conocimiento y actitud en prevención de trabajadores lesionados de una empresa metalmecánica en México. Salud de los Trabajadores. 2009; 17(1):49-57.

9. Cortes, José. Seguridad e higiene en el trabajo: Técnicas de prevención accidentes laborales. Madrid: Ed. TEBAR, 2007.

10. Pérez F, Costa Morería J, Meneses Rodríguez C. Percepción de Riesgo de los Agricultores con Respecto a los Pesticidas en un Área Agrícola del Estado de Rio de Janeiro, Brasil. Ciencia y Trabajo. 2007;9(26):164-171.

11. Zamorano González, Benito, PARRA SIERRA, Víctor, PENA CARDENAS, Fabiola et al. Conocimiento y actitud en prevención de trabajadores lesionados de una empresa metalmecánica en México. Salud de los Trabajadores, jun. 2009, vol.17, no.1, p.49-57. ISSN 1315-0138.

12. Boon G, Mercado A. Automatización flexible en la industria. Difusión y producción de máquinas-herramienta de control numérico en América Latina. Ciudad de México: Limusa; 1990.

13. Diagnóstico sobre Clima Organizacional y Accidentalidad en la empresa colombiana. Santa fé de Bogotá: Departamento Tecnico Suratep; 2008.

14. Saari J. Accidentes y Gestión de la Seguridad: Prevención de Accidentes. 3rd ed. Madrid: Enciclopedia de la Organización Internacional del Trabajo; 2011.

15. Soler, F. Curso básico Cyclops de Prevención de Riesgos Laborales. Riesgos específicos y su prevención en el sector del Metal. Mutual Cyclops, Mutua de Accidentes del Trabajo y Enfermedades Profesionales de la Seguridad Social no 126; 2000. 